\title{
CUIDADOS RELACIONADOS À DERMATITE NA ÁREA DE FRALDAS EM IDOSOS HOSPITALIZADOS
}

\section{CARE RELATED TO DIAPER RASH IN HOSPITALIZED ELDERY}

\section{CUIDADOS RELACIONADOS A DERMATITE EN EL ÁREA DE PAÑALES EN ANCIANOS HOSPITALIZADOS}

\author{
Jéssica Luana Gomes Silva ${ }^{1}$, Andrea Mathes Faustino².
}

\section{RESUMO}

Objetivo: identificar a ocorrência da dermatite na área de fraldas em idosos hospitalizados. Método: trata-se de um estudo descritivo, transversal, com abordagem quantitativa e epidemiológica. O estudo foi desenvolvido em unidade de Clínica Médica de um hospital universitário, localizado no Distrito Federal, Brasil e foi utilizado um instrumento de coleta de dados com as seguintes variáveis: características sociodemográficas, morbidades associadas, hábitos de vida e cuidados realizados diante da dermatite na área de fraldas, além do exame físico da pele na região da genitália e áreas adjacentes. A amostra foi composta por 40 idosos. Resultados: em relação à ocorrência da dermatite na área de fraldas, $42 \%$ dos idosos apresentaram a alteração na pele, $55 \%$ realizavam a troca de fraldas pelo menos a cada 4horas ou menos; quanto à frequência da higiene íntima, $60 \%$ a realizavam uma vez ao dia, sendo que 32\% realizavam esta limpeza somente utilizando água. Conclusão: foi possível verificar que uma parcela significativa dos idosos internados na unidade de clínica médica, utilizando fraldas e/ou dispositivos para controle de incontinência, desenvolveram a dermatite na área de fraldas, gerando a necessidade de um diagnóstico precoce por parte da equipe multiprofissional e medidas de baixa complexidade para a prevenção do problema.

Descritores: Dermatite das fraldas; Incontinência urinária; Idoso.

\begin{abstract}
Objective: to identify the occurrence of dermatitis in diaper areas in hospitalized elderly. Method: this is a descriptive, crosssectional study with a quantitative and epidemiological approach. The study was carried out in a Clinical Medical Unit of a university hospital, located in the Federal District, Brazil, and a data collection instrument was used. It has the following variables: sociodemographic characteristics, associated morbidities, life habits, and care given to dermatitis in diaper areas, in addition to the physical examination of the skin on and around the genital area. The sample consisted of 40 elderly people. Results: in relation to the occurrence of dermatitis in diaper areas, $42 \%$ of the elderly had a change in their skin, $55 \%$ performed diaper changes at least every 4 hours or less; regarding to the intimate hygiene frequency, $60 \%$ performed it once a day; and $32 \%$ performed it using only water. Conclusion: a significant proportion of the elderly hospitalized in the medical clinic, wearing diapers and/or using incontinence control devices, developed diaper dermatitis, generating the need for an early diagnosis by the multiprofessional team and low complexity measures to prevent the problem.
\end{abstract}

Keywords: Diaper rash; Urinary incontinence; Elderly.

\section{RESUMEN}

Objetivo: identificar la ocurrencia de la dermatitis en el área de pañales en ancianos hospitalizados. Método: se trata de un estudio descriptivo, transversal, con abordaje cuantitativo y epidemiológico. El estudio fue desarrollado en unidad de Clínica Médica de un hospital universitario, localizado en el Distrito Federal, Brasil y se utilizó un instrumento de recolección de datos con las siguientes variables: características sociodemográficas, morbilidades asociadas, hábitos de vida y cuidados realizados frente a la dermatitis en el área de pañales, además del examen físico de la piel en la región de los genitales y áreas adyacentes. La muestra fue compuesta por 40 ancianos. Resultados: en relación a la ocurrencia de la dermatitis en el área de pañales, $42 \%$ de los ancianos presentaron la alteración en la piel, 55\% realizaban el cambio de pañales por lo menos cada 4 horas o menos; en cuanto a la frecuencia de la higiene íntima, el 60\% la realizaban una vez al año día, siendo que el 32\% realizaba esta limpieza solamente utilizando agua. Conclusión: fue posible verificar que una parte significativa de los ancianos internados en la unidad de clínica médica, utilizando pañales y/o dispositivos para control de incontinencia desarrollaron la dermatitis en el área de pañales, generando la necesidad de un diagnóstico precoz por parte del equipo multiprofesional y medidas de baja complejidad para la prevención del problema. Descriptores: Dermatitis del Pañal; Incontinencia urinaria; Anciano.

${ }^{1}$ Graduada em Enfermagem pelo Departamento de Enfermagem da Faculdade de Ciências da Saúde, Universidade de Brasília. ${ }^{2}$ Graduada em Enfermagem. Especialista em Gerontologia, Doutora em Ciências da Saúde, Docente na Universidade de Brasília.

Como citar este artigo:

Silva JLG, Faustino AM. Cuidados Relacionados A Dermatite Na Área De Fraldas Em Idosos Hospitalizados. Revista de Enfermagem do Centro Oeste Mineiro. 2019;9:e2721. [Access___ Available in:___ DOI: http://dx.doi.org/10.19175/recom.v9i0.2721 


\section{INTRODUÇÃO}

O processo de envelhecimento humano traz consigo vários desafios para o cuidado, aumentando a necessidade de direcionamento a fim de promover uma assistência em todos os níveis de complexidade. Dentre os desafios, pode-se destacar a incontinência urinária (IU) como um problema de saúde significante para a população idosa, sendo considerada uma condição que afeta tanto os aspectos físicos, quanto os aspectos psicológicos dessa parcela populacional ${ }^{1}$.

A IU pode ser definida como a situação na qual ocorre perda involuntária de urina, constituindo um problema social e higiênico, além de constituir-se em um importante problema de repercussão econômica, podendo gerar impactos na qualidade de vida do indivíduo acometido ${ }^{2}$.

Estima-se que 50 milhões de pessoas sofram com a IU no mundo, sendo mais comum em mulheres, nas suas diferentes fases de vida, podendo acometer até $50 \%$ da população feminina, principalmente aquelas com mais de 60 anos de idade ${ }^{3}$. Estudos apontam que a IU em idosos apresenta prevalência média de 30\% naqueles que vivem na comunidade, de 40 a $70 \%$ entre os hospitalizados, e em torno de $50 \%$ daqueles que vivem em Instituições de Longa Permanência (ILPI) ${ }^{4}$. Um estudo epidemiológico realizado na cidade de São Paulo com 2143 idosos teve como resultado uma prevalência de IU de $26,2 \%$ entre as mulheres, concluindo que esta condição está relacionada com o avançar da idade e com as limitações funcionais consequentes ${ }^{5}$.

A IU tem uma etiologia habitualmente multifatorial. Fatores físicos, cognitivos, funcionais e psicossociais contribuem para o aumento da possibilidade de o indivíduo ser acometido por esse problema ${ }^{6}$.

Além disso, a IU pode ser causada por qualquer fator que faça com que a pressão intravesical seja maior que a pressão uretral máxima. A pressão intravesical pode estar aumentada em decorrência da hiperatividade vesical, causando incontinência de urgência, ou por distensão vesical, resultando na condição de incontinência por extravasamento ${ }^{6-7}$. Além das complicações psicossociais, inclui-se o sentimento de vergonha, isolamento social, piora na qualidade de vida e surgimento de quadros depressivos. A IU também predispõe o indivíduo à infecções do trato urinário, lesão por pressão e o aparecimento de dermatites perineais ${ }^{(4,8)}$. Alterações de pele associada à IU podem ser de difícil manejo, pois necessitam do controle de vários fatores como por exemplo: o microclima da pele, processo de cicatrização, bem como do próprio cuidado quanto à periodicidade das trocas de fralda ou de outro dispositivo urinário para o controle da perdas urinárias.

Uma variedade de termos tem sido utilizada para descrever lesões de pele associadas à IU, tais como: dermatite perineal, erupção cutânea por uso de fralda, dermatite irritativa de fraldas, dermatite amoniacal, dermatite associada à incontinência, dermatite da área das fraldas, entre outros ${ }^{4}$.

Para fins desta pesquisa, utilizaremos o termo dermatite da área das fraldas (DAF), definido como diferentes dermatoses inflamatórias, de etiologia multifatorial, que atinge a área do corpo coberta pela fralda, afetando normalmente a região do períneo, região anogenital, região glútea, abdômen inferior e coxas. A DAF pode ser interpretada como o resultado final de simultâneos eventos e induzidas por exposição a diversos fatores, tais como: fezes, urina, hidratação, friç̧ão, temperatura, irritantes químicos e microorganismos $^{9}$. A etiologia e a patogênese da DAF são pouco compreendidas, mas sabe-se que a maceração, oclusão, presença de bactérias e Cândida são condições importantes a serem consideradas $^{10}$. Essas situações estão diretamente relacionadas ao cuidado com a pele de pessoas que fazem uso de fraldas, associadas a medidas preventivas em relação ao desenvolvimento de lesões de pele.

Quando a DAF acomete um indivíduo idoso, é necessário considerar que a pele deste indivíduo apresenta diversas alterações decorrentes do processo de envelhecimento ${ }^{9}$. Com a perda do tecido de sustentação, gordura subcutânea, menor estímulo sensitivo, diminuição de pelos e glândulas sudoríparas e sebáceas, a população idosa é mais propensa a desencadear lesões na pele e mais susceptíveis a adquirir infecções ${ }^{11}$.

As profundas alterações da pele, decorrentes do processo de envelhecimento, acarretam a maior fragilidade cutânea e diminuição da capacidade da pele em atuar como barreira protetora, aumentando assim o risco de desenvolver lesões na pele $\mathrm{e}^{12}$. Em idosos hospitalizados, as alterações na pele podem estar mais comprometidas, pois estarão associadas às 
condições de saúde pelas quais este indivíduo foi hospitalizado, além de outros agravos associados à baixa mobilidade (restrição ao leito, como por exemplo), lesão por pressão, ressecamento, atrito, entre outros problemas.

Devido aos poucos estudos sobre o problema da DAF na população idosa, e pelo fato de que a maioria das vezes, as medidas de prevenção e cuidados serem realizadas pela equipe de enfermagem ${ }^{9}$, justifica-se realizar tal estudo, o qual teve, por objetivo, identificar a ocorrência da dermatite na área de fraldas (DAF) em idosos hospitalizados.

\section{MÉTODOS}

Trata-se de estudo descritivo, transversal, com abordagem quantitativa e epidemiológica. A investigação epidemiológica tem por objetivo fornecer medidas precisas da ocorrência das doenças ou outros desfechos, sendo que o seu alvo

é sempre uma população humana, que pode ser definida em termos geográficos ou outro qualquer $^{13}$.

O estudo foi desenvolvido em uma unidade de Clínica Médica de um hospital universitário, localizado no Distrito Federal, Brasil. Esta unidade era composta por duas alas, com trinta e uma enfermarias no total, sendo cada uma delas compostas de 2 a 4 leitos. Durante o período da coleta, haviam 67 leitos funcionantes na unidade. O período da coleta de dados foi entre janeiro a junho de 2017.

A população foi composta por idosos, ou seja, pessoas com 60 anos ou mais, internadas nesta unidade, que possuíam incontinência urinária, sendo a amostra não probabilística. Os critérios de inclusão adotados na pesquisa foram: ser idoso, pessoas com 60 anos ou mais; possuir incontinência urinária; utilizar dispositivos para o controle da incontinência urinária do tipo: fralda, absorvente higiênico, roupa íntima descartável, entre outros. A amostra final foi composta por 40 idosos.

A coleta de dados foi realizada em uma única etapa, por meio do uso de um instrumento elaborado pelas pesquisadoras, por meio do qual foram coletados dados quanto a: aspectos sociodemográficos, necessidades de cuidados, capacidade funcional para as atividades básicas de vida diária, hábitos de vida e cuidados realizados diante da DAF. Além disso, foi realizado o exame físico da pele e região da genitália e áreas adjacentes, com o intuito de verificar a ocorrência da DAF.

Para a organização dos dados, as informações coletadas foram transcritas para uma planilha do programa Microsoft Excel, na qual houve uma codificação e revisão para validar os dados digitados. Assim, posteriormente, foi realizada a análise estatística descritiva, na qual os dados coletados foram organizados e categorizados.

A pesquisa atendeu às normas dispostas na Resolução 466/2012, do Conselho Nacional de Saúde, e foi aprovada no Comitê de Ética em Pesquisa, da Faculdade de Ciências da Saúde da Universidade de Brasília, sob o $n^{\circ}$ CAAE: 60936116.9.0000.0030.

\section{RESULTADOS E DISCUSSÃO}

Em relação à idade, os idosos incluídos no estudo estavam com 60 até 92 anos, sendo a maioria na faixa etária entre 70 e 79 anos de idade (42\%). A média de idade foi de 75,85 anos, $60 \%$ eram homens e quanto à cor autodeclarada, $58 \%$ se consideravam pardos. Quanto ao grau de instrução, $90 \%$ dos entrevistados eram alfabetizados, $42 \%$ eram casados, com renda familiar entre 1 e 3 salários mínimos 77\% (Tabela 1).

Considerando uma série de fatores relacionados à idade e que afetam a eficiência da barreira protetora da pele, percebe-se um aumento no risco de DAF na pessoa idosa. Concomitantemente, existe um maior risco de incontinência urinária e fecal nessa faixa etária, levando a uma maior incidência de DAF entre os idosos, comparados aos pacientes mais jovens ${ }^{14}$.

Pesquisas mostram que a prevalência da DAF foi apontada como responsável por $7 \%$ das lesões de pele em pacientes incontinentes internados em casa de repouso, e $42 \%$ em adultos incontinentes e hospitalizados, resultado semelhante ao observado no presente estudo, onde $42 \%$ dos idosos internados na clínica médica, utilizando fraldas e/ou outros dispositivos para incontinência haviam desenvolvido a DAF ${ }^{4}$.

Com relação à especialidade médica da internação, $40 \%$ estavam internados pela especialidade geriatria $(40 \%)$ e, em segundo lugar, pela oncologia (32\%). Dentre os entrevistados, $42 \%$ possuíam DAF, sendo que $12 \%$ relataram que os primeiros sintomas surgiram com menos de uma semana de uso da fralda (Tabela 1). 
Quanto à necessidade de ter um cuidador para auxiliar nas atividades de autocuidado, $92 \%$ dos idosos possuía um, pois $87 \%$ relatou depender de ajuda para a realização de atividades básicas de vida diária (Tabela 1 ).

Tabela 1. Distribuição dos dados quanto aos aspectos sociodemográficos, necessidades de cuidados, capacidade funcional para as atividades básicas de vida diária em idosos hospitalizados $(n=40)$. Brasília (DF), 2017.

\begin{tabular}{|c|c|c|}
\hline Variáveis & $\mathbf{n}$ & $\%$ \\
\hline \multicolumn{3}{|l|}{ Sexo } \\
\hline Feminino & 16 & 40,0 \\
\hline Masculino & 24 & 60,0 \\
\hline \multicolumn{3}{|c|}{ Idade dos idosos (em anos) } \\
\hline 60 a 69 & 10 & 25,0 \\
\hline 70 a 79 & 17 & 42,5 \\
\hline 80 a 89 & 11 & 27,5 \\
\hline 90 a 99 & 2 & 5,0 \\
\hline \multicolumn{3}{|l|}{ Etnia } \\
\hline Branco & 7 & 17,5 \\
\hline Negro & 9 & 22,5 \\
\hline Pardo & 23 & 57,5 \\
\hline Indígena & 1 & 2,5 \\
\hline \multicolumn{3}{|l|}{ Grau de Instrução } \\
\hline Alfabetizados & 17 & 42,5 \\
\hline Analfabetos & 3 & 7,5 \\
\hline Ensino Médio & 10 & 25,0 \\
\hline Ensino Fundamental & 7 & 17,5 \\
\hline Ensino Superior & 2 & 5,0 \\
\hline Não soube responder & 1 & 2,5 \\
\hline \multicolumn{3}{|l|}{ Estado Civil } \\
\hline Casado & 17 & 42,5 \\
\hline Solteiro & 5 & 12,5 \\
\hline Viúvo & 14 & 35,0 \\
\hline Divorciado & 2 & 5,0 \\
\hline União Estável & 2 & 5,0 \\
\hline \multicolumn{3}{|l|}{ Renda Família } \\
\hline Até 1 salário & 2 & 5,0 \\
\hline 1 a 3 salários & 31 & 77,5 \\
\hline 3 a 6 salários & 3 & 7,5 \\
\hline Acima de 6 salários & 4 & 10,0 \\
\hline \multicolumn{3}{|l|}{ Especialidade Médica } \\
\hline Geriatria & 16 & 40,0 \\
\hline Oncologia & 13 & 32,5 \\
\hline Pneumologia & 3 & 7,5 \\
\hline Gastroenterologia & 3 & 7,5 \\
\hline Outras & 5 & 12,5 \\
\hline \multicolumn{3}{|l|}{ Possui DAF } \\
\hline Sim & 17 & 42,5 \\
\hline Não & 23 & 57,5 \\
\hline \multicolumn{3}{|c|}{ Quando surgiram os primeiros sintomas } \\
\hline Menos de uma semana & 5 & 12,5 \\
\hline Mais de uma semana & 10 & 25,0 \\
\hline Mais de um mês & 2 & 5,0 \\
\hline Não se aplica & 23 & 57,5 \\
\hline \multicolumn{3}{|c|}{ Tem ou Precisa de Cuidador } \\
\hline $\operatorname{Sim}$ & 37 & 92,5 \\
\hline Não & 3 & 7,5 \\
\hline \multicolumn{3}{|c|}{ Atividades básicas de vida diária } \\
\hline Dependente & 35 & 87,5 \\
\hline Independente & 2 & 5,0 \\
\hline Parcial & 3 & 7,5 \\
\hline Total & 40 & 100,0 \\
\hline
\end{tabular}

Fonte: Dados da pesquisa, 2017. 
Dentre os idosos entrevistados, $42 \%$ ingeriam menos de um litro de água por dia. Quanto aos cuidados relacionados à DAF, 55\% realizavam a troca de fraldas pelo menos a cada 4 horas ou em menor tempo. No que se refere à frequência da higiene íntima, 60\% realizava uma vez ao dia e $32 \%$ executava esta limpeza utilizando somente água. Quanto à temperatura da água, para a higiene, 98\% utilizava água morna (Tabela 2).

Sabe-se que as alterações da pele, provenientes da DAF, são desencadeadas por uma combinação de diversos fatores, sendo os mais significativos, o contato prolongado ou a irritação através da urina e fezes, além da maceração produzida pela umidade e o calor no local. Assim, o contato prolongado com a fralda molhada por urina e lípases fecais, pode intensificar a probabilidade do desenvolvimento de alterações na pele. Considerando esta realidade, reconhece-se a importância da troca frequente da fralda descartável associada à higiene íntima adequada, com o intuito de prevenir o desenvolvimento da DAF e a possível proliferação de bactérias que podem causar infecções secundárias pela instalação da lesão cutânea $^{14}$.

Percebe-se, através dos resultados obtidos no presente estudo, que grande parte dos entrevistados permanece com a mesma fralda por cerca de 4 a 8 horas, o que pode aumentar o risco de estes pacientes desencadearem uma lesão ou dificultando o restabelecimento de uma lesão já existente.

Tabela 2. Distribuição dos dados quanto a hábitos de vida e cuidados realizados diante da DAF em idosos hospitalizados ( $n=40)$. Brasília (DF), 2017.

\begin{tabular}{|c|c|c|}
\hline Variáveis & $\mathbf{n}$ & $\%$ \\
\hline \multicolumn{3}{|l|}{ Hábitos de vida - Ingestão de água } \\
\hline Menos de 1 litro & 17 & 42,5 \\
\hline De 1 a 2 litros & 22 & 55,0 \\
\hline Mais que 2 litros & 1 & 2,5 \\
\hline \multicolumn{3}{|l|}{ Troca de fraldas ou dispositivo } \\
\hline Pelo menos a cada 2 horas ou menos & 2 & 5,0 \\
\hline Pelo menos a cada 4 horas ou menos & 22 & 55,0 \\
\hline Pelo menos a cada 8 horas ou menos & 14 & 35,0 \\
\hline Mais de 8 horas & 2 & 5,0 \\
\hline \multicolumn{3}{|l|}{ Frequência de higiene íntima } \\
\hline Uma vez ao dia & 24 & 60,0 \\
\hline Duas vezes ao dia & 12 & 30,0 \\
\hline Três vezes ao dia & 1 & 2,5 \\
\hline Quatro vezes ao dia ou mais & 3 & 7,5 \\
\hline \multicolumn{3}{|l|}{ Como é realizada a limpeza } \\
\hline Apenas com água & 13 & 32,5 \\
\hline Com água e sabonete & 27 & 67,5 \\
\hline \multicolumn{3}{|c|}{ Temperatura da água utilizada para higiene } \\
\hline Quente & 1 & 2,5 \\
\hline Morna & 39 & 97,5 \\
\hline \multicolumn{3}{|l|}{ Uso de pomada/cremes de barreira } \\
\hline Sim & 22 & 55,0 \\
\hline Não & 18 & 45,0 \\
\hline \multicolumn{3}{|l|}{ Uso de hidratante na pele } \\
\hline Mais de uma vez ao dia & 8 & 20,0 \\
\hline Pelo menos uma vez ao dia & 21 & 52,5 \\
\hline Mais de três vezes por semana & 7 & 17,5 \\
\hline Pelos menos uma vez por semana & 3 & 7,5 \\
\hline Não usa & 1 & 2,5 \\
\hline \multicolumn{3}{|l|}{ Tipo de sabonete } \\
\hline Sabonete neutro & 21 & 52,5 \\
\hline Sabonete Antisséptico & 8 & 20,0 \\
\hline Sabonete Hidratante & 10 & 25,0 \\
\hline Outro & 1 & 2,5 \\
\hline
\end{tabular}




\begin{tabular}{|c|c|c|}
\hline Variáveis & $\mathrm{n}$ & $\%$ \\
\hline \multicolumn{3}{|l|}{ Materiais utilizados para higiene íntima } \\
\hline Esponjas / Buchas & 9 & 22,5 \\
\hline Não utiliza & 26 & 65,0 \\
\hline Outros & 5 & 12,5 \\
\hline \multicolumn{3}{|l|}{ Uso de outro Dispositivo } \\
\hline Sim & 19 & 47,5 \\
\hline Não & 21 & 52,5 \\
\hline \multicolumn{3}{|l|}{ Tipo de fralda ou dispositivo } \\
\hline Fraldas descartáveis & 21 & 52,5 \\
\hline Fralda + Sonda vesical de demora & 14 & 35,0 \\
\hline Fralda + Dispositivo urinário externo & 4 & 10,0 \\
\hline Roupa íntima descartável & 1 & 2,5 \\
\hline Total & 40 & 100,0 \\
\hline
\end{tabular}

Fonte: Dados da pesquisa, 2017.

É importante destacar que os cuidados de higiene devem ser realizados com maior frequência, evitando assim a exposição ao $\mathrm{pH}$ alcalino. Além disso, destaca-se que a limpeza deve ser realizada com água e sabão, seguida de um creme protetor ${ }^{14}$, do tipo barreira protetora. Ao contrário do que é recomendado na literatura, maior parte da amostra (60\%) realiza a limpeza da pele somente uma vez por dia, e apenas $2 \%$ da amostra realiza a limpeza três vezes ou mais por dia. Contudo, a maioria quando realiza a limpeza, o faz com água e sabão (67\%).

Em relação ao tipo de sabão utilizado para limpeza, é importante destacar que produtos antibacterianos e/ou antissépticos não são recomendados, pois podem ressecar a pele, aumentar $\mathrm{o} \mathrm{pH}$ e contribuir para erosão da epiderme, sendo mais indicado a utilização de sabão hidratante ou neutro ${ }^{9}$. Há poucas evidências na literatura, mas, em alguns estudos, já se destaca a eficácia de produtos de limpeza que possam ser deixados na pele, ou seja, sem enxágue, pois o uso do método de limpeza com água e sabão seria menos indicado quando já houver a presença da dermatite ${ }^{15}$.

Quanto ao uso de pomadas e/ou cremes de barreira protetora, $55 \%$ utilizavam algum tipo de produto, $52 \%$ faziam uso de hidratante corporal pelo menos uma vez ao dia. No que diz respeito ao tipo de sabonete utilizado para banho e/ou limpeza íntima, 52\% utilizavam sabonete neutro e $20 \%$ sabonete antisséptico. Quanto a materiais para a realização da higiene íntima, $65 \%$ não utilizavam qualquer tipo de material, sendo que $22 \%$ utilizavam buchas ou esponjas para tal (Tabela 2).

A aplicação de pomadas ou cremes de barreira protetora podem e devem ser utilizados como forma de prevenção ou para tratamento da DAF. Alguns estudos realizados para avaliar as alterações das funções da pele com dermatite, durante o tratamento com a pasta de óxido de zinco e vaselina, demonstraram que a pasta reduziu a força de atrito da pele com a fralda, além de reduzir o risco de infecções por fungos $^{9,15}$. Foi observado, no presente estudo, que o uso de pomadas com barreira de proteção, pelos idosos, era realizado, na maioria das vezes, como medida de tratamento por aqueles que já apresentavam a DAF, e não como forma de prevenção.

No que diz respeito ao uso de hidratante corporal, $52 \%$ da amostra fazia uso pelo menos uma vez por dia. O comprometimento da elasticidade cutânea somada ao ressecamento proveniente da diminuição da secreção das glândulas sebáceas e sudoríparas leva a pessoa idosa a uma maior exposição e risco aumentado para o rompimento cutâneo. Logo, a hidratação da pele é uma medida preventiva que ajuda amenizar o ressecamento da pele, diminuindo o risco de lesões ${ }^{16}$. Além disso, estes hidratantes devem ser aplicados suavemente com movimentos circulares, evitando massagear as áreas de proeminência óssea ou que estejam hiperemiadas, como forma preventiva para o surgimento de outras lesões ${ }^{17}$.

Todos os pacientes entrevistados faziam uso de fraldas ou roupa íntima descartável, sendo que $47 \%$ também faziam uso de outro dispositivo para controle da incontinência urinária. Quanto ao tipo de fraldas ou dispositivo urinário, 52\% utilizava fraldas descartáveis, seguidos de $35 \%$ que associavam fraldas descartáveis ao uso da sonda vesical de demora (Tabela 2).

Fatores mecânicos como a friç̧ão e cisalhamento são alguns dos responsáveis pelo surgimento da DAF. Estudos apontam que a população idosa tem uma menor tolerância a friç̧ão e a pressão, acarretando um maior risco 
para dermatites ${ }^{4}$. Logo, a utilização de materiais, como buchas e esponjas, para a realização da limpeza íntima, deve ser evitada, diminuindo a chance do aparecimento ou agravamento de lesões pelo atrito que esses materiais podem causar na pele.

\section{CONSIDERAÇÕES FINAIS}

O estudo permitiu verificar a ocorrência da DAF em idosos hospitalizados, bem como as condições de assistência prestados por seus cuidadores. Pôde ser observado que uma parcela significativa dos idosos internados na unidade de clínica médica, utilizando fraldas e/ou dispositivos para controle de incontinência possuía DAF, gerando um desafio importante para a equipe multiprofissional de saúde.

Entende-se a necessidade de um diagnóstico precoce, com vistas a prevenção de lesões, exigindo da equipe de saúde que presta os cuidados a esses idosos, a implementação de ações que possam contribuir para a melhoria das condições de saúde e qualidade de vida, com base nas melhores práticas de cuidados, baseadas em evidências científicas, as quais foram apresentadas no presente estudo.

O estudo apresenta limitações ao apresentar a realidade de um único cenário, com características específicas, não sendo possível a generalização dos resultados encontrados. Ainda assim, o estudo traz contribuições importantes ao evidenciar a situação de muitos idosos que utilizam fraldas devido à IU em situação de hospitalização, que podem ter chance elevada de desenvolver lesões de pele. Destarte, medidas simples de trocas de fraldas, higiene e hidratação da pele, uso de cremes do tipo barreira protetora podem ser estratégias eficazes para a prevenção da DAF, bem como das complicações associadas. Além disso, é importante destacar que estratégias de orientação e educação em saúde aos pacientes e seus cuidadores têm forte impacto no cuidado prestado e na prevenção do problema, o que pode ser feito durante o preparo para alta hospitalar, com vistas à continuidade do cuidado no domicílio.

\section{REFERÊNCIAS}

1 - Locks MOH, Santos SMA. Uso de fralda geriátrica em hospitais: Solução ou problema? Estima 2015;13(1):27-34. DOI: 10.5327/Z1806$\underline{3144201500010006}$

2 - Carvalho MP, Andrade FP, Peres W, Martinelli $\mathrm{T}$, Simch F, Orcy RB et al. O impacto da incontinência urinária e seus fatores associados em idosas. Rev Bras Geriatr Gerontol. 2014;17(4):721-30. DOI: $10.1590 / 1809-$ 9823.2014.13135

3 - Henkes DF, Fiori A, Carvalho JAM, Tavares KO, Frare JC. Incontinência urinária: $O$ impacto na vida de mulheres acometidas e o significado do tratamento fisioterapêutico. Semin, Ciênc Biol Saúde 2015;36(2):45-56. DOI: 10.5433/16790367.2015v36n2p45

4 - Cunha CV, Nascimento DFD, Felix F, Cunha P, Pena LHG. Dermatite associada à incontinência em idosos: Caracterização, prevenção e tratamento. Estima 2015 [citado 20 dez 2017]; 13(3). Available in: https://www.revistaestima.com.br/index.php/est ima/article/view/218

5 - Tamanini JTN, Lebrão ML, Duarte YAO, Santos JLF, Laurenti R. Analysis of the prevalence of and factors associated with urinary incontinence among elderly people in the Municipality of São Paulo, Brazil: SABE Study (Health, Wellbeing and Aging). Cad Saúde Pública 2009;25(8):1756-62. DOI: 10.1590/S0102-311X2009000800011

6 - Pitangui ACR, Silva RG, Araújo RC. Prevalência e impacto da incontinência urinária na qualidade de vida de idosas institucionalizadas. Rev Bras Geriatr Gerontol. 2012;15(4):619-26. DOI: 10.1590/S1809-98232012000400002

7 - Faria CA, Menezes AMN, Rodrigues AO, Ferreira ALV, Bolsas CN. Incontinência urinária e noctúria: Prevalência e impacto sobre qualidade de vida em idosas numa Unidade Básica de Saúde. Rev Bras Geriatr Gerontol. 2014 17(1): 17$25 . \quad$ DOI: $10.1590 /$ S180998232014000100003

8 -Chianca TCM, Gonçales PC, Salgado PO, Machado BO, Amorim GL, Alcoforado CLGC. Dermatite associada à incontinência: Estudo de coorte em pacientes críticos. Rev. gaúch. enferm. 2016;37(nesp):1-9. DOI: 10.1590/19831447.2016.esp.68075

9 -Aquino AL, Chianca TCM, Brito RCS. Integridade da pele prejudicada, evidenciada por dermatite da área das fraldas: Revisão integrativa. Rev Eletr Enferm. 2012; 14(2):414-24. DOI: 10.5216/ree.v14i2.14977

10 - Fernandes JD, Machado MCR, Oliveira ZNP. Fisiopatologia da dermatite da área das fraldas: Parte I. An Bras Dermatol. 2008;83(6):567-71. DOI: $10.1590 /$ S0365-05962008000600012

11 - Bliss DZ, Funk T, Jacobson M, Savik K. Incidence and characteristics of incontinence associated dermatitis in community-dwelling 
persons with fecal incontinence. J Wound Ostomy Continence Nurs. 2015;42(5):525-30. DOI: 10.1097/WON.0000000000000159

12 - Cavalcante AMRZ, Moreira A, Azevedo KB, Lima LR, Coimbra WKAM. Diagnóstico de enfermagem: Integridade tissular prejudicada identificado em idosos na Estratégia de Saúde da Família. Rev Eletr Enferm. 2010;12(4):727-35. DOI: $10.5216 /$ ree.v12i4.8425

13 - Oliveira MRF. Métodos epidemiológicos: Uso e aplicações pelos serviços de saúde. Epidemiol Serv Saúde 2010;19(2):89-90. DOI: 10.5123/S1679-49742010000200001

14 - Rosa NM, Inoue KC, Silvino MCS, Oliveira MLF. Treatment for dermatites associated with incontinence in institutionalized elderly: Integrative review. Rev RENE 2013 [citado 20 dez 2017]; 14(5):1031-40. Disponível em: http://www.revistarene.ufc.br/revista/index.php/ revista/article/viewFile/1188/pdf

15 - Beeckman D, Van Damme N, Schoonhoven L, Van Lancker A, Kottner J, Beele $\mathrm{H}$ et al. Interventions for preventing and treating incontinence-associated dermatitis in adults. Cochrane Database Syst Rev. 2016;11(11): CD011627. DOI: 10.1002/14651858.CD011627. pub2/epdf

16 - Fortes TML, Suffredini IB. Avaliação de pele em idoso: Revisão da literatura. J Health Sci Inst. 2014 [citado 5 dez 2017]; 32(1):94-101. Disponível em:

https://www.unip.br/comunicacao/

publicacoes/ics/edicoes/2014/01 jan-mar/V32 n1 2014 p94a101.pdf

17 - Ferreira JDL, Aguiar ESS, Lima CL, Brito KKG, Costa MML, Soares MJGO. Ações preventivas para úlcera por pressão em idosos com declínio funcional de mobilidade física no âmbito domiciliar. Estima 2016;14(1):36-42. DOI: $\underline{10.5327 / Z 1806-3144201600010006}$

Nota: Este artigo faz parte do Trabalho de Conclusão de Curso de Enfermagem da Universidade de Brasília (UnB), intitulado: "Dermatite na área de fraldas em idosos internados em unidade de clínica médica".

Recebido em: 05/12/2018

Aprovado em: 25/03/2019

Endereço de correspondência:

Andréa Mathes Faustino

Universidade de Brasília

Campus Universitário Darcy Ribeiro, Faculdade de Ciências

da Saúde, Departamento de Enfermagem/Sala 05

CEP: 70910900 - Brasília/DF - Brasil
E- mail: andreamathes@unb.br 